\title{
Extended Hubbard Model in the Dimer Representation. II. Lattice Hamiltonian in the Large $U$ Limit
}

\author{
B. Grabiec and M. Matlak* \\ Institute of Physics, Silesian University \\ Uniwersytecka 4, 40-007 Katowice, Poland
}

(Received December 14, 2001)

\begin{abstract}
Using the exact decomposition of the sc lattice into a set of interacting dimers (each dimer is described by the extended Hubbard Hamiltonian) and exact solution of the dimer problem (preceding paper) we exactly find the form of the extended Hubbard model in the case of a crystal in the large $U$ limit. We apply a new, nonperturbative approach based on the exact projection procedure onto a dimer subspace occupied by electrons in this limit (it is the only assumption). The resulting Hamiltonian is very complicated and contains a variety of multiple magnetic and nonmagnetic interactions deeply hidden in its original form (site representation). We also present a simplified version of the model to better visualize a mixture of different interactions resulting from this approach.
\end{abstract}

PACS numbers: $71.10 .-\mathrm{w}, 71.10 . \mathrm{Ca}, 71.10 . \mathrm{Fd}$

\section{Introduction}

The tendency of electrons to avoid each other is very well known in the theory of strongly correlated electron systems (see e.g. Refs. [1, 2] for a review). This tendency, identifying in this way strongly correlated systems, can be expressed by the condition $U \gg W(U-$ intrasite Coulomb repulsion, $W-$ band width of the conduction band). In the limit $U \gg W$ (large $U$ limit) the second-order perturbation theory applied to such systems leads to the well-known $t-J$ model

*corresponding author; e-mail: matlak@server.phys.us.edu.pl 
(see Ref. [3]) describing indirect exchange interaction between electron spins located on different lattice sites. The popularity of the $t-J$ model has enormously increased in recent years after the suggestion, Ref. [4], that the electronic properties of high- $T_{\mathrm{C}}$ superconductors can be described with the use of this model (see e.g. Refs. $[2,5]$ for a review and papers cited therein). This fact strongly enhances the importance of the $t-J$ model in the physics of strongly correlated electron systems.

The $t-J$ model can be derived by applying perturbation expansion or canonical transformation (see Refs. $[3,6-10]$ and also $[2,5]$ for a review) to the Hubbard Hamiltonian, Ref. [11], where one takes into account that in the strong correlation limit $W / U$ is very small. In other words, each of these methods is equivalent to the perturbation expansion with respect to $W / U$. It is, however, possible to propose quite general approach and to derive the resulting Hamiltonian in the large $U$ limit without using perturbation expansion and perform the calculation for a more general case of extended Hubbard model (see preceding paper [12]). In Ref. [12] we have presented the exact decomposition of the original extended Hubbard model for the sc lattice (it can be generalized to any lattice, too) into a set of interacting dimers where each dimer problem has been exactly solved (see (12) in Ref. [12]). The method has been tested in Ref. [12] for a dimer (the smallest complex of interacting atoms), described by the extended Hubbard model (see (3) in Ref. [12]). The dimer energy spectrum consists of 16 energies, 6 of them in the large $U$ limit take on large, positive values (see (12) in Ref. [12]), much larger than the other. It means that the mentioned 6 levels, producing negligible small terms in the partition function cannot be occupied by electrons and therefore we can reject them from the considerations in the large $U$ limit. Taking this into account we could rewrite the dimer Hamiltonian in the large $U$ limit (see (22) in Ref. [12]) and after introducing Hubbard operators and spin operators (see (23), (24) in Ref. [12]) we were able to present the dimer Hamiltonian in the second quantization. The final form of this Hamiltonian is nothing else but a generalization of the $t-J$ model (see e.g. Ref. [3]) resulting from the extended Hubbard model (see (28) in Ref. [12]). It is important to stress that we have obtained this result in a nonperturbative way using only the reduction of the full dimer space (16 eigenvectors) to the subspace of 9 states, occupied by electrons in the large $U$ limit. We have also shown that exactly the same result (see Sec. 4 in Ref. [12]) can also be derived with the use of the projection technique (projection onto the occupied dimer states). A further generalization of this technique for the case of a crystal is given in the present paper. The resulting extended Hubbard Hamiltonian in the large $U$ limit, obtained in the nonperturbative way, is very complicated. It shows, however, in an explicit way a structure of many multiple magnetic, "quasi-magnetic" and nonmagnetic, competitive interactions, deeply hidden in the original version of the model. 


\section{Extended Hubbard model in the large $U$ limit}

In the preceding paper [12] we exactly solved the eigenvalue problem of the Hubbard dimer which allowed to express the dimer Hamiltonian in the form

$$
H^{\mathrm{D}}=\sum_{\alpha} E_{\alpha}\left|E_{\alpha}\right\rangle\left\langle E_{\alpha}\right|
$$

where $E_{\alpha}$ and $\left|E_{\alpha}\right\rangle$ were given by the expressions (12) in Ref. [12]. After excluding from the considerations unoccupied dimer energy levels $\left(E_{22}, E_{23}, E_{31}, E_{32}\right.$, $\left.E_{33}, E_{34}, E_{4}\right)$ we derived the second quantization form of the dimer Hamiltonian (denoted by $\bar{H}^{\mathrm{D}}$ ) in the large $U$ limit (see (28) in Ref. [12]). We obtained exactly the same result with the use of the projection technique (see Sec. 4 in Ref. [12]). A similar approach, after several modifications, can be applied in the case of a crystal (sc lattice). The extended Hubbard Hamiltonian (see (2) in Ref. [12]) can also be written in a similar form to (1). We can write

$$
H=\sum_{\gamma} \bar{E}_{\gamma}\left|\bar{E}_{\gamma}\right\rangle\left\langle\bar{E}_{\gamma}\right|
$$

where, in contrary to $(1)$, the energies $\bar{E}_{\gamma}$ and $\left|\bar{E}_{\gamma}\right\rangle$ are unknown. We can, however, expand the eigenvectors $\left|\bar{E}_{\gamma}\right\rangle$ into the series of the dimer eigenvectors (cf. (12) in Ref. [12])

$$
\left|\bar{E}_{\gamma}\right\rangle=\sum_{\gamma_{1}, \ldots, \gamma_{M}} c_{\gamma_{1}, \ldots, \gamma_{M}}^{\gamma}\left|E_{\gamma_{1}}\right\rangle \ldots\left|E_{\gamma_{M}}\right\rangle
$$

assuming that the crystal consists of $M$ dimers. Inserting (3) into (2) we get

$$
H=\sum_{\gamma} E_{\gamma} \sum_{\substack{\gamma_{1}, \ldots, \gamma_{M} \\ \gamma_{1}^{\prime}, \ldots, \gamma_{M}^{\prime}}} c_{\gamma_{1}, \ldots, \gamma_{M}}^{\gamma} c_{\gamma_{1}^{\prime}, \ldots, \gamma_{M}^{\prime}}^{* \gamma}\left|E_{\gamma_{1}}\right\rangle \ldots\left|E_{\gamma_{M}}\right\rangle\left\langle E_{\gamma_{1}^{\prime}}\right| \ldots\left\langle E_{\gamma_{M}^{\prime}}\right| .
$$

It is clear that to obtain the extended Hubbard Hamiltonian for large $U$ we have to project (4) onto the subspace of the lower lying dimer states with the use of the projection operator

$$
P=P_{1} P_{2} \ldots P_{M}
$$

where $P_{I}$ is given by (32) or (33) in Ref. [12] (the omitted in Ref. [12] dimer index $I=1,2, \ldots, M$ should actually be added). Similarly to (34) in [12] we denote the extended Hubbard Hamiltonian in the large $U$ limit by $\bar{H}$. Thus, we define

$$
\bar{H}=P H P
$$

and we use $H$ in the form given by the expression (2) in Ref. [12]. Taking into account that $P^{2}=P\left(P_{I}^{2}=P_{I},\left[P_{I}, P_{J}\right]=0\right)$ we obtain 


$$
\begin{aligned}
\bar{H}= & P\left[\sum_{I} \bar{H}_{I}^{\mathrm{D}}-t \sum_{I, \sigma}\left(\bar{c}_{I, 2, \sigma}^{+} \bar{c}_{I+1,1, \sigma}+\bar{c}_{I+1,1, \sigma}^{+} \bar{c}_{I, 2, \sigma}\right)\right. \\
& -t \sum_{I \neq J, \sigma}\left(\bar{c}_{I, 1, \sigma}^{+} \bar{c}_{J, 1 \sigma}+\bar{c}_{I, 2, \sigma}^{+} \bar{c}_{J, 2, \sigma}\right)+J^{(1)} \sum_{I, \sigma} \overline{\bar{n}}_{I, 2, \sigma} \overline{\bar{n}}_{I+1,1, \sigma} \\
& +\frac{J^{(1)}}{2} \sum_{I, J, \sigma}\left(\overline{\bar{n}}_{I, 1, \sigma} \overline{\bar{n}}_{J, 1, \sigma}+\overline{\bar{n}}_{I, 2, \sigma} \overline{\bar{n}}_{J, 2, \sigma}\right)+J^{(2)} \sum_{I, \sigma} \overline{\bar{n}}_{I, 2, \sigma} \overline{\bar{n}}_{I+1,1,-\sigma} \\
& \left.+\frac{J^{(2)}}{2} \sum_{I, J, \sigma}\left(\overline{\bar{n}}_{I, 1, \sigma} \overline{\bar{n}}_{J, 1,-\sigma}+\overline{\bar{n}}_{I, 2, \sigma} \overline{\bar{n}}_{J, 2,-\sigma}\right)\right] \equiv P \overline{\bar{H}}
\end{aligned}
$$

The operators $\bar{c}_{I, \alpha, \sigma}\left(\bar{c}_{I, \alpha, \sigma}^{+}\right)$have the same meaning as (36) in Ref. [12] and the corresponding expressions for them (after adding the dimer index $I$ ) are given by (37), (38) therein. The operators $\overline{\bar{n}}_{I, \alpha, \sigma}$ in $(7)$ are defined by the relation

$$
\overline{\bar{n}}_{I, \alpha, \sigma}=P_{I} n_{I, \alpha, \sigma} P_{I} .
$$

Introducing Hubbard operators and spin operators (see (23) and (24) in Ref. [12]) we can find

$$
\begin{aligned}
\bar{n}_{I, 1, \uparrow} & =S_{I, 1}^{z}\left(1-\frac{n_{I, 2}^{b}}{2}\right)+\frac{n_{I, 1}^{a}}{2}\left(1-\frac{n_{I, 2}^{b}}{2}\right)+\beta\left[S_{I, 1}^{z} S_{I, 2}^{z}-\frac{n_{I, 1}^{a} n_{I, 2}^{a}}{4}\right. \\
+ & S_{I, 1}^{+} S_{I, 2}^{-}+S_{I, 1}^{-} S_{I, 2}^{+}-n_{I, 2}^{a} S_{I, 1}^{z}+n_{I, 1}^{a} S_{I, 2}^{z}+\frac{n_{I, 1}^{b}}{4}\left(1-n_{I, 2}^{a}\right)+\frac{n_{I, 2}^{b}}{4}\left(1-n_{I, 1}^{a}\right) \\
& -\frac{1}{4} n_{I, 1}^{b} n_{I, 2}^{b}+\frac{1}{2}\left(b_{I, 1, \uparrow}^{+} a_{I, 1, \downarrow}^{+} a_{I, 2, \downarrow} b_{I, 2, \uparrow}+b_{I, 2, \uparrow}^{+} a_{I, 2, \downarrow}^{+} a_{I, 1, \downarrow} b_{I, 1, \uparrow}\right) \\
& \left.+\frac{1}{2}\left(a_{I, 1, \uparrow}^{+} a_{I, 2, \downarrow}^{+} a_{I, 1, \downarrow} a_{I, 2, \uparrow}+a_{I, 2, \uparrow}^{+} a_{I, 1, \downarrow}^{+} a_{I, 2, \downarrow} a_{I, 1, \uparrow}\right)\right] \\
& +\delta\left(a_{I, 1,1}^{+} b_{I, 2, \uparrow}+a_{I, 2, \downarrow}^{+} b_{I, 1, \downarrow}+b_{I, 2, \uparrow}^{+} a_{I, 1, \uparrow}+b_{I, 1, \downarrow}^{+} a_{I, 2, \downarrow}\right), \\
\overline{\bar{n}}_{I, 1, \downarrow} & =-S_{I, 1}^{z}\left(1-\frac{n_{I, 2}^{b}}{2}\right)+\frac{n_{I, 1}^{a}}{2}\left(1-\frac{n_{I, 2}^{b}}{2}\right)+\beta\left[S_{I, 1}^{z} S_{I, 2}^{z}-\frac{n_{I, 1}^{a} n_{I, 2}^{a}}{4}\right. \\
+ & S_{I, 1}^{+} S_{I, 2}^{-}+S_{I, 1}^{-} S_{I, 2}^{+}+n_{I, 2}^{a} S_{I, 1}^{z}-n_{I, 1}^{a} S_{I, 2}^{z}+\frac{n_{I, 1}^{b}}{4}\left(1-n_{I, 2}^{a}\right)+\frac{n_{I, 2}^{b}}{4}\left(1-n_{I, 1}^{a}\right) \\
& -\frac{1}{4} n_{I, 1}^{b} n_{I, 2}^{b}+\frac{1}{2}\left(b_{I, 1, \uparrow}^{+} a_{I, 1, \downarrow}^{+} a_{I, 2, \downarrow} b_{I, 2, \uparrow}+b_{I, 2, \uparrow}^{+} a_{I, 2, \downarrow}^{+} a_{I, 1, \downarrow} b_{I, 1, \uparrow}\right) \\
& \left.+\frac{1}{2}\left(a_{I, 1, \uparrow}^{+} a_{I, 2, \downarrow}^{+} a_{I, 1, \downarrow} a_{I, 2, \uparrow}+a_{I, 2, \uparrow}^{+} a_{I, 1, \downarrow}^{+} a_{I, 2, \downarrow} a_{I, 1, \uparrow}\right)\right] \\
+ & \delta\left(a_{I, 1, \downarrow}^{+} b_{I, 2, \downarrow}+a_{I, 2, \uparrow}^{+} b_{I, 1, \uparrow}+b_{I, 2, \downarrow}^{+} a_{I, 1, \downarrow}+b_{I, 1, \uparrow}^{+} a_{I, 2, \uparrow}\right) .
\end{aligned}
$$


The expression for $\overline{\bar{n}}_{I, 2, \sigma}(\sigma=\uparrow, \downarrow)$ can easily be obtained by replacing $1 \leftrightarrow 2$ in (9) and (10). The coefficients $\beta$ and $\delta$ are given by (42) in Ref. [12]. To obtain the second quantization form of $\overline{\bar{H}}$ (see (7)) we should insert (37) and (38) from Ref. [12], as well as (9) and (10) into (7). In this way we obtain the second quantization form of $\overline{\bar{H}}$ in the large $U$ limit. It is, in principle, possible to write down the explicit, final form for the Hamiltonian $\overline{\bar{H}}$. The formula, however, is unfortunately too long to present it here (too many terms resulting from many multiplications in (7)). Therefore we restrict ourselves only to indicate the existence of a variety of different interactions, contributing to $\overline{\bar{H}}$ and to present a simplified version of this Hamiltonian in the next section. It is easy to see that, generally, $\overline{\bar{H}}$ contains the terms independent of $\beta$ and $\delta$, however, the terms proportional to $\beta, \delta, \beta^{2}, \delta^{2}$ and $\beta \delta$ appear also in the final form for $\overline{\bar{H}}$. Because the operators $\bar{c}_{I, \alpha, \sigma}\left(\bar{c}_{I, \alpha, \sigma}^{+}\right)$and $\overline{\bar{n}}_{I, \alpha, \sigma}(\alpha=1,2 ; \sigma=\uparrow, \downarrow)$ contain spin operators (see (37), (38) in Ref. [12] and (9), (10) (present paper)) the resulting Hamiltonian $\overline{\bar{H}}$ consists of many magnetic (ferromagnetic, antiferromagnetic) and more complex interactions competing one with another. The structure of the total Hamiltonian $\bar{H}$ in the large $U$ limit that we are interested in (see (6) and (7)) is much more complicated due to the presence of the projector $P$ (see (5)) on the left hand side of (7). The operator $P$ itself is a product of all $P_{I}$ projectors (see (33) in Ref. [12]). It makes the structure of the total Hamiltonian $\bar{H}$ much more complex, resulting in sums of products of many multiple magnetic and nonmagnetic, competitive interactions, deeply hidden in the original version of the extended Hubbard model, written in the Wannier representation (see (1) in Ref. [12]). We have obtained this result using the exact dimer representation of the construction operators (see (37), (38) in Ref. [12]) and (9), (10) (present paper) in the large $U$ limit. The presence of a variety of many multiple magnetic and nonmagnetic interactions in the resulting Hamiltonian $\bar{H}$ (see (7)) has very strong, final consequences. It entirely explains how difficult and delicate is the problem to find the thermodynamical properties of the model in the approximate way (the exact solution of the model does not exist till now). The use of the most sophisticated methods does not remove a danger to overestimate the role of some important interactions and to underestimate the other, producing in this way artifacts. It is the reason why the resulting thermodynamical properties of the extended Hubbard model so strongly depend on the quality of applied approximations.

\section{Simplified version of the model in the large $U$ limit}

The enormously high complexity of the model (7), presented in Sec. 2, can easily be demonstrated in a more explicit way. To do it, we perform simplifications, described beneath. We can e.g. apply the Taylor expansion with respect to two small parameters $x=t / U$ and $x^{(2)}=J^{(2)} / U$ in the expressions (37) and (38) 
from Ref. [12], as well as in (9) and (10) (present paper). It seems to be, however, more reasonable to choose another way. Starting with typical values for the band width $W=1 \mathrm{eV}$ and $U=5 \mathrm{eV}$ we can see that $x=\frac{1}{60}(W=12 t$ for the $\mathrm{sc}$ lattice). Assuming that $J^{(2)}=0.1 \mathrm{eV}$ we obtain $x^{(2)}=\frac{1}{50}$. Thus, introducing $x=\frac{1}{60}$ and $x^{(2)}=\frac{1}{50}$ into the expressions for $\beta$ and $\delta$ (see (42) in Ref. [12]) we can find $\beta \approx 0.00058$ and $\delta \approx 0.017 \approx 30 \beta$. In other words we can neglect the terms proportional to $\beta$ in (37) and (38) of Ref. [12] and also in (9) and (10) excluding also double occupations $\left(n_{I, 1(2)}^{b} \approx 0\right)$ in the large $U$ limit (see (30a) in Ref. [12]). The same can be said about the operators $\underline{\underline{a}}_{I, 1(2), \sigma}$ and $\underline{a}_{I, 1(2), \sigma}$ in $(41 \mathrm{a}, \mathrm{b})$ of Ref. [12] where we neglect the terms proportional to $\beta$, and we put again $n_{I, 1(2)}^{b} \approx 0$ in (41b) of Ref. [12]. Taking into account that $\delta^{2} \ll \delta$ we can consequently perform all the calculations, concerning $\overline{\bar{H}}$ and $\bar{H}$ (see (7)) to obtain the final result as a linear form in $\delta$ (neglecting all the terms proportional to $\beta, \beta^{2}, \delta^{2}$ and $\beta \delta$ ). Using this approach we first find the simplified form for $\bar{H}_{I}^{\mathrm{D}}$ (see (28) in Ref. [12]) and $P_{I}$ (see (33) in Ref. [12]). We obtain

$$
\bar{H}_{I}^{\mathrm{D}}=\bar{H}_{I}^{\mathrm{D}}(0)+\delta \bar{H}_{I}^{\mathrm{D}}(1)
$$

where

$$
\begin{aligned}
& \bar{H}_{I}^{\mathrm{D}}(0)=-t \sum_{\sigma}\left(a_{I, 1, \sigma}^{+} a_{I, 2, \sigma}+a_{I, 2, \sigma}^{+} a_{I, 1, \sigma}\right)+2 J^{(1)}\left(S_{I, 1}^{z} \cdot S_{I, 2}^{z}+\frac{n_{I, 1}^{a} n_{I, 2}^{a}}{4}\right) \\
& \quad-2 J^{(2)}\left(S_{I, 1}^{z} \cdot S_{I, 2}^{z}-\frac{n_{I, 1}^{a} n_{I, 2}^{a}}{4}\right) \\
& \bar{H}_{I}^{\mathrm{D}}(1)=\frac{4 t}{1-x^{(2)}}\left(S_{I, 1} \cdot S_{I, 2}-\frac{n_{I, 1}^{a} n_{I, 2}^{a}}{4}\right)+J^{(2)} Q_{I} \\
& Q_{I}=\sum_{\alpha=1,2} \sum_{\sigma}\left(a_{I, \alpha, \sigma}^{+} b_{I, \bar{\alpha}, \sigma}+b_{I, \alpha, \sigma}^{+} a_{I, \bar{\alpha}, \sigma}\right),
\end{aligned}
$$

and

$$
P_{I}=1+\delta Q_{I}
$$

Similarly to (11) we can decompose $\overline{\bar{H}}$ (see (7)) when neglecting the terms proportional to $\beta$ in (37) and (38) of Ref. [12], as well as in (9) and (10). Applying this procedure we get

$$
\overline{\bar{H}}=\overline{\bar{H}}(0)+\delta \overline{\bar{H}}(1)
$$

where 


$$
\begin{aligned}
& \overline{\bar{H}}(0)=\sum_{I} \bar{H}_{I}^{\mathrm{D}}(0)-t \sum_{I, \sigma}\left(a_{I, 2, \sigma}^{+} a_{I+1,1, \sigma}+a_{I+1,1, \sigma}^{+} a_{I, 2, \sigma}\right)-t \sum_{I \neq J, \sigma} \sum_{\alpha=1,2} a_{I, \alpha, \sigma}^{+} a_{J, \alpha, \sigma} \\
& +2 J^{(1)} \sum_{I}\left(S_{I, 2}^{z} \cdot S_{I+1,1}^{z}+\frac{n_{I, 2}^{a} n_{I+1,1}^{a}}{4}\right)-2 J^{(2)} \sum_{I}\left(S_{I, 2}^{z} \cdot S_{I+1,1}^{z}-\frac{n_{I, 2}^{a} n_{I+1,1}^{a}}{4}\right) \\
& +J^{(1)} \sum_{I \neq J, \sigma} \sum_{\alpha=1,2}\left(S_{I, \alpha}^{z} \cdot S_{J, \alpha}^{z}+\frac{n_{I, \alpha}^{a} n_{J, \alpha}^{a}}{4}\right) \\
& -J^{(2)} \sum_{I \neq J, \sigma} \sum_{\alpha=1,2}\left(S_{I, \alpha}^{z} \cdot S_{J, \alpha}^{z}-\frac{n_{I, \alpha}^{a} n_{J, \alpha}^{a}}{4}\right), \\
& \overline{\bar{H}}(1)=\sum_{I} \bar{H}_{I}^{\mathrm{D}}(1)-t \sum_{I, \sigma}\left(a_{I, 2, \sigma}^{+} \bar{a}_{I+1,1, \sigma}+\bar{a}_{I, 2, \sigma}^{+} a_{I+1,1, \sigma}+a_{I+1,1, \sigma}^{+} \bar{a}_{I, 2, \sigma}\right. \\
& \left.+\bar{a}_{I+1,1, \sigma}^{+} a_{I, 2, \sigma}\right)-t \sum_{I \neq J, \sigma} \sum_{\alpha=1,2}\left(a_{I, \alpha, \sigma}^{+} \bar{a}_{J, \alpha, \sigma}+\bar{a}_{I, \alpha, \sigma}^{+} a_{J, \alpha, \sigma}\right) \\
& +t \sum_{I} S_{I+1,1}^{z} \cdot\left[\left(a_{I, 2, \uparrow}^{+} a_{I+1,2, \uparrow}-a_{I, 2, \downarrow}^{+} a_{I+1,2, \downarrow}\right)+\left(a_{I+1,2, \uparrow}^{+} a_{I, 2, \uparrow}-a_{I+1,2, \downarrow}^{+} a_{I, 2, \downarrow}\right)\right] \\
& +t \sum_{I} S_{I, 2}^{z} \cdot\left[\left(a_{I+1,1, \uparrow}^{+} a_{I, 1, \uparrow}-a_{I+1,1, \downarrow}^{+} a_{I, 1, \downarrow}\right)+\left(a_{I, 1, \uparrow}^{+} a_{I+1,1, \uparrow}-a_{I, 1, \downarrow}^{+} a_{I+1,1, \downarrow}\right)\right] \\
& +t \sum_{I}\left[S_{I+1,1}^{-} \cdot\left(a_{I, 2, \uparrow}^{+} a_{I+1,2, \downarrow}+a_{I+1,2, \uparrow}^{+} a_{I, 2, \downarrow}\right)+S_{I, 2}^{-} \cdot\left(a_{I, 1, \uparrow}^{+} a_{I+1,1, \downarrow}+a_{I+1,1, \uparrow}^{+} a_{I, 1, \downarrow}\right)\right] \\
& +t \sum_{I}\left[S_{I+1,1}^{+} \cdot\left(a_{I+1,2, \downarrow}^{+} a_{I, 2, \uparrow}+a_{I, 2, \downarrow}^{+} a_{I+1,2, \uparrow}\right)+S_{I, 2}^{+} \cdot\left(a_{I+1,1, \downarrow}^{+} a_{I, 1, \uparrow}+a_{I, 1, \downarrow}^{+} a_{I+1,1, \uparrow}\right)\right] \\
& +t \sum_{I \neq J} \sum_{\alpha=1,2}\left\{S_{I, \alpha}^{z} \cdot\left[\left(a_{I, \bar{\alpha}, \uparrow}^{+} a_{J, \alpha, \uparrow}-a_{I, \bar{\alpha}, \downarrow}^{+} a_{J, \alpha, \downarrow}\right)+\left(a_{J, \alpha, \uparrow}^{+} a_{I, \bar{\alpha}, \uparrow}-a_{J, \alpha, \downarrow}^{+} a_{I, \bar{\alpha}, \downarrow}\right)\right]\right\} \\
& +t \sum_{I \neq J} \sum_{\alpha=1,2}\left\{S_{I, \alpha}^{-} \cdot\left(a_{I, \bar{\alpha}, \uparrow}^{+} a_{J, \alpha, \downarrow}+a_{J, \alpha, \uparrow}^{+} a_{I, \bar{\alpha}, \downarrow}\right)+S_{I, \alpha}^{+} \cdot\left(a_{I, \bar{\alpha}, \downarrow}^{+} a_{J, \alpha, \uparrow}+a_{J, \alpha, \downarrow}^{+} a_{I, \bar{\alpha}, \uparrow}\right)\right\} \\
& +\frac{\left(J^{(1)}+J^{(2)}\right)}{2} \sum_{I, \sigma} \sum_{\alpha=1,2}\left[n_{I, 2}^{a}\left(a_{I+1, \alpha, \sigma}^{+} b_{I+1, \bar{\alpha}, \sigma}+b_{I+1, \bar{\alpha}, \sigma}^{+} a_{I+1, \alpha, \sigma}\right)\right. \\
& \left.+n_{I+1,1}^{a}\left(a_{I, \bar{\alpha}, \sigma}^{+} b_{I, \alpha, \sigma}+b_{I, \alpha, \sigma}^{+} a_{I, \bar{\alpha}, \sigma}\right)\right]+2\left(J^{(1)}-J^{(2)}\right) \sum_{I}\left[S _ { I , 2 } ^ { z } \cdot \left(s_{I+1,1,2}^{z}-s_{I+1,2,1}^{z}\right.\right. \\
& \left.\left.+\bar{s}_{I+1,2,1}^{z}-\bar{s}_{I+1,1,2}^{z}\right)+S_{I+1,1}^{z} \cdot\left(s_{I, 2,1}^{z}-s_{I, 1,2}^{z}+\bar{s}_{I, 1,2}^{z}-\bar{s}_{I, 2,1}^{z}\right)\right]+\frac{\left(J^{(1)}+J^{(2)}\right)}{2} \\
& \times \sum_{I \neq J, \sigma} \sum_{\alpha=1,2} n_{I, \alpha}^{a}\left(a_{J, \alpha, \sigma}^{+} b_{J, \bar{\alpha}, \sigma}+b_{J, \bar{\alpha}, \sigma}^{+} a_{J, \alpha, \sigma}+a_{J, \bar{\alpha}, \sigma}^{+} b_{J, \alpha, \sigma}+b_{J, \alpha, \sigma}^{+} a_{J, \bar{\alpha}, \sigma}\right) \\
& +2\left(J^{(1)}-J^{(2)}\right) \sum_{I \neq J} \sum_{\alpha=1,2} S_{I, \alpha}^{z}\left(s_{J, \alpha, \bar{\alpha}}^{z}-s_{J, \bar{\alpha}, \alpha}^{z}+\bar{s}_{\boldsymbol{J}, \bar{\alpha}, \alpha}^{z}-\bar{s}_{J, \alpha, \bar{\alpha}}^{z}\right),
\end{aligned}
$$

where 


$$
\begin{aligned}
& s_{I, \alpha, \bar{\alpha}}^{z}=\frac{1}{2}\left(a_{I, \alpha, \uparrow}^{+} b_{I, \bar{\alpha}, \uparrow}-a_{I, \alpha, \downarrow}^{+} b_{I, \bar{\alpha}, \downarrow}\right), \\
& \bar{s}_{I, \alpha, \bar{\alpha}}^{z}=\left(s_{I, \alpha, \bar{\alpha}}^{z}\right)^{+}=\frac{1}{2}\left(b_{I, \alpha, \uparrow}^{+} a_{I, \bar{\alpha}, \uparrow}-b_{I, \alpha, \downarrow}^{+} a_{I, \bar{\alpha}, \downarrow}\right), \\
& \bar{a}_{I, 1(2), \sigma}=\underline{b}_{I, 2(1), \sigma}+a_{I, 2(1),-\sigma}^{+} a_{I, 1(2),-\sigma} b_{I, 1(2), \sigma}+a_{I, 2(1), \sigma} \frac{n_{I, 1(2)}^{a}}{2},
\end{aligned}
$$

and $\underline{b}_{I, 1(2), \sigma}$ is given by (41b) in Ref. [12] where we put $n_{1(2)}^{b} \approx 0$ in the large $U$ limit and $\bar{\alpha}=1$ (2) if $\alpha=2$ (1). We see that in spite of applying approximate procedure the formula for the Hamiltonian $\overline{\bar{H}}$, given by $(16),(17)$ and (18) is very complicated and very long. It contains, however, all terms independent of $\delta$ and proportional to $\delta$. The simplified form of the extended Hubbard model in the large $U$ limit (7) that we are interested in is given by

$$
\bar{H}=P \overline{\bar{H}} \approx \prod_{L=1}^{M}\left(1+\delta Q_{L}\right) \overline{\bar{H}} \approx \overline{\bar{H}}(0)+\delta\left[\sum_{L=1}^{M} Q_{L} \overline{\bar{H}}(0)+\overline{\bar{H}}(1)\right],
$$

where we have again retained only the terms independent of $\delta$ and proportional to $\delta$. The formulae for $Q_{L}, \overline{\bar{H}}(0)$, and $\overline{\bar{H}}(1)$ are given by (14), (17), and (18), respectively. The simplified form of the extended Hubbard model in the large $U$ limit (20) shows a variety of different magnetic and nonmagnetic interactions appearing in $\overline{\bar{H}}(0), \sum_{L=1}^{M} Q_{L} \overline{\bar{H}}(0)$ and $\overline{\bar{H}}(1)$ when looking at (17), (14) and (18). We can find Ising-type interactions, Heisenberg-type interactions and also more complex Heisenberg interactions (see (17), (18)) where the localized spin operator is multiplied by a term similar to spin operator which "transports" electron spin from one lattice site to the other (as e.g. the fourth and next few terms in (18)), producing in this way "quasi-magnetic" interactions. All of these interactions, present in the simplified form of the Hamiltonian (20) will be of a much more complex nature when one includes to $\overline{\bar{H}}$ (see (16)) all terms proportional to $\beta, \delta^{2}$, and $\beta \delta$, as mentioned in Sec. 2. The projection operator $P$ in this case (see (5)) cannot be generally written in such a simple form as (15) because each $P_{I}$ (see (33) in Ref. [12]) contains also magnetic interactions. It produces in the total expression for $\bar{H}=P \overline{\bar{H}}$ (see (7)) sums of products of many multiple (magnetic, "quasi-magnetic", etc.) interactions of enormously high complexity.

\section{Conclusions}

We have shown a new and straightforward way how to derive the extended Hubbard Hamiltonian for the sc lattice in the strong correlations limit based on the dimer decomposition of the original lattice into a set of interacting dimers using the exact solution of each dimer problem. The strong correlation limit is taken into account by the reduction of the space of the dimer states to the subspace corresponding to the occupied energy levels. The method can be, in principle, applied 
to another models to derive the resulting Hamiltonian in the strong correlation limit. The only restriction for a further popularization of the method can be the complexity of laborious calculations exponentially growing up in the case when instead of dimers we will use more complex clusters (trimers, etc.).

\section{References}

[1] F. Gebhard, The Mott Metal-Insulator Transition, Springer Tracts in Modern Physics, Vol. 137, Springer, Berlin 1997.

[2] Yu.A. Izyumov, Phys. Usp. 40, 445 (1997).

[3] P.W. Anderson, Phys. Rev. 115, 2 (1959).

[4] P.W. Anderson, Science 235, 1196 (1997).

[5] E. Dagotto, Rev. Mod. Phys. 66, 763 (1994).

[6] A.B. Harris, R.V. Lange, Phys. Rev. 157, 295 (1967).

[7] K.A. Chao, J. Spałek, A.M. Oleś, J. Phys. C 10, L271 (1977); Phys. Status Solidi B 84, 747 (1977); Phys. Rev. B 18, 3453 (1978).

[8] J. Spałek, A.M. Oleś, Physica B 86-88, 375 (1977).

[9] J. Spałek, K.A. Chao, J. Phys. C 13, 5241 (1980).

[10] C. Gros, R. Joynt, T.M. Rice, Phys. Rev. B 36, 381 (1987).

[11] J. Hubbard, Proc. R. Soc. Lond. A 276, 238 (1963); A 281, 401 (1964).

[12] B. Grabiec, M. Matlak, Acta Phys. Pol. A 101, (2002). 